\title{
Method for constructing an equivalent mechanical model of an elastic system of a machine with various technological equipment
}

\author{
Sergey L. Vasilevykh ${ }^{1, *}$, Alexandr V. Udalov ${ }^{1}$, and Evgeniy S. Shelihov ${ }^{2}$ \\ ${ }^{1}$ Vyatka State University, Kirov, Russian Federation \\ 2 Orenburg State University, Orenburg, Russian Federation
}

\begin{abstract}
The article deals with the construction of an equivalent mechanical model of the elastic system of the machine for turning nonrigid shafts with various technological equipment, including machining in centers and machining in a chuck with the rear rotating center being clamped without using additional support and using a vibration-damper lunette. The relevance of this study due to the fact that in view of low stiffness processed non-rigid shaft technology system machine-tool-toolworkpiece is extremely sensitive to the deformations caused by the action of cutting forces. As a result, significant processing errors are generated. High sensitivity to deformations serves as a source of intensive vibrations of the workpiece, which leads to deterioration of the quality of the machined surface of the part, a decrease in the durability and durability of the cutting tool, accelerated wear and loss of guaranteed machine accuracy parameters. This makes it essential to significantly reduce the cutting conditions, to resort to multi-pass processing, which greatly increases the laboriousness and cost of manufacturing products. In this context, the aim of this study - the creation of an equivalent mechanical model of the machine, allowing the process of studies to determine the boundaries of sustainable non-rigid turning shafts considering the different ways of mounting the workpiece on the machine. The materials of the article can be useful for engineers and scientists in the field of engineering.
\end{abstract}

\section{Introduction}

In general, the considered equivalent elastic system of the machine-tool-tool-workpiece, interacting with the cutting system, is characterized by the following basic properties affecting its output parameters: accuracy of adjustment, geometrical accuracy, rigidity, thermophysical characteristic, wear resistance, vibration resistance, elasticity. These properties of an equivalent elastic system determine its ability to resist the action of the main disturbance, and the deviations of these properties from the nominal values cause corresponding processing errors.

As a result of the analysis of the connections that arise in the processing system under consideration, the dominant factors that prevent the intensification of the processing of

\footnotetext{
${ }^{*}$ Corresponding author: sergei.vasiljevyh@yandex.ru
} 
details of low rigidity are established: errors in setting of the basic elements of the machine, deformation of the workpiece under the influence of cutting force and vibration of the workpiece, resulting in cutting process, which lead to corresponding errors in shape of the part, and also the undulation of the treated surface.

As a result of the review and analysis of known works [1-13], it has been established that the work connected with the study of the effect on the stability of the machine-tooltool-workpiece system of the technological equipment used has not been carried out, and the few works that are available are based mainly on experimental research.

Thus, the following research objectives were identified:

1. Justify the choice of the main elements of the dominant vibrational elastic system of the machine in the processing of non-rigid shaft.

2. Develop an equivalent mechanical model for studying the vibration resistance of turning non-rigid shafts.

3. Obtain boundary conditions for all types of technological equipment of the machine.

\section{Materials and Methods}

The study used the materials and methods of the theory of cutting, strength of materials, as well as mathematical modeling.

\section{Selection of the design model of the elastic system of the machine with various technological equipment}

As a result of experimental studies of the process of turning non-rigid shafts, it is established that the main oscillatory elements of the MTTW system under consideration are either the shaft itself (if the shaft mass is smaller in comparison with the saddle mass) or the shaft with the saddle and the slide (if the shaft mass is comparable or greater than the weight of the saddle).

Thus, in the equivalent mechanical model (calculation scheme) of the machine, the following dominant elements must be taken into account: a machined shaft with a saddle and a slide, dynamic cutting forces and various technological adjustments.

Due to the fact that processing of non-rigid long $(l / d \geq 20)$ shafts is considered, it is also necessary to take into account the continuous distribution of the shaft mass along its length $[9,11]$.

We assume that the axis of symmetry of the undeformed shaft coincides with the $x$ axis.

In general, the shaft section has three possible displacements: transverse, longitudinal and torsional.

The spectra of the natural frequencies of the transverse, longitudinal and torsional oscillations of the shaft fixed in the centers have the following form:

$$
\begin{gathered}
f_{\text {jnon }}=\frac{\pi^{2} \cdot j^{2}}{4 l}\left(\frac{d}{l}\right) \sqrt{\frac{E}{\rho}}, \\
f_{\text {jкруm }}=\frac{\pi^{2} \cdot j}{l} \sqrt{\frac{G}{\rho}}, \\
f_{\text {jпрод }}=\frac{\pi^{2} \cdot j}{l} \sqrt{\frac{E}{\rho}},
\end{gathered}
$$


where $p$ - specific weight of the material, $l$ - shaft length, $d$ - shaft diameter, $E$ и $G$ tensile and shear modulus, respectively.

From the practice of processing non-rigid shafts it is known that the excitation of vibrations usually occurs at frequencies close to the lowest natural frequencies of free transverse oscillations of the shaft. Consequently, when studying the excitation of vibrations of a nonrigid shaft, one can confine ourselves to studying the dominant transverse oscillations of the shaft. Therefore, only transverse vibrations of the shaft $V(x, t)$ and $W(x, t)$ in two orthogonal planes $(y, x)$ and $(z, x)$, respectively, are taken into account in the equivalent mechanical model (Fig. 1). The right coordinate system $x y z$ is chosen so that the $x$-axis coincides with the axis of symmetry of the undeformed shaft axis $y$ - with the direction of infeed, and $z$ axis - orthogonal axes $x, y$.
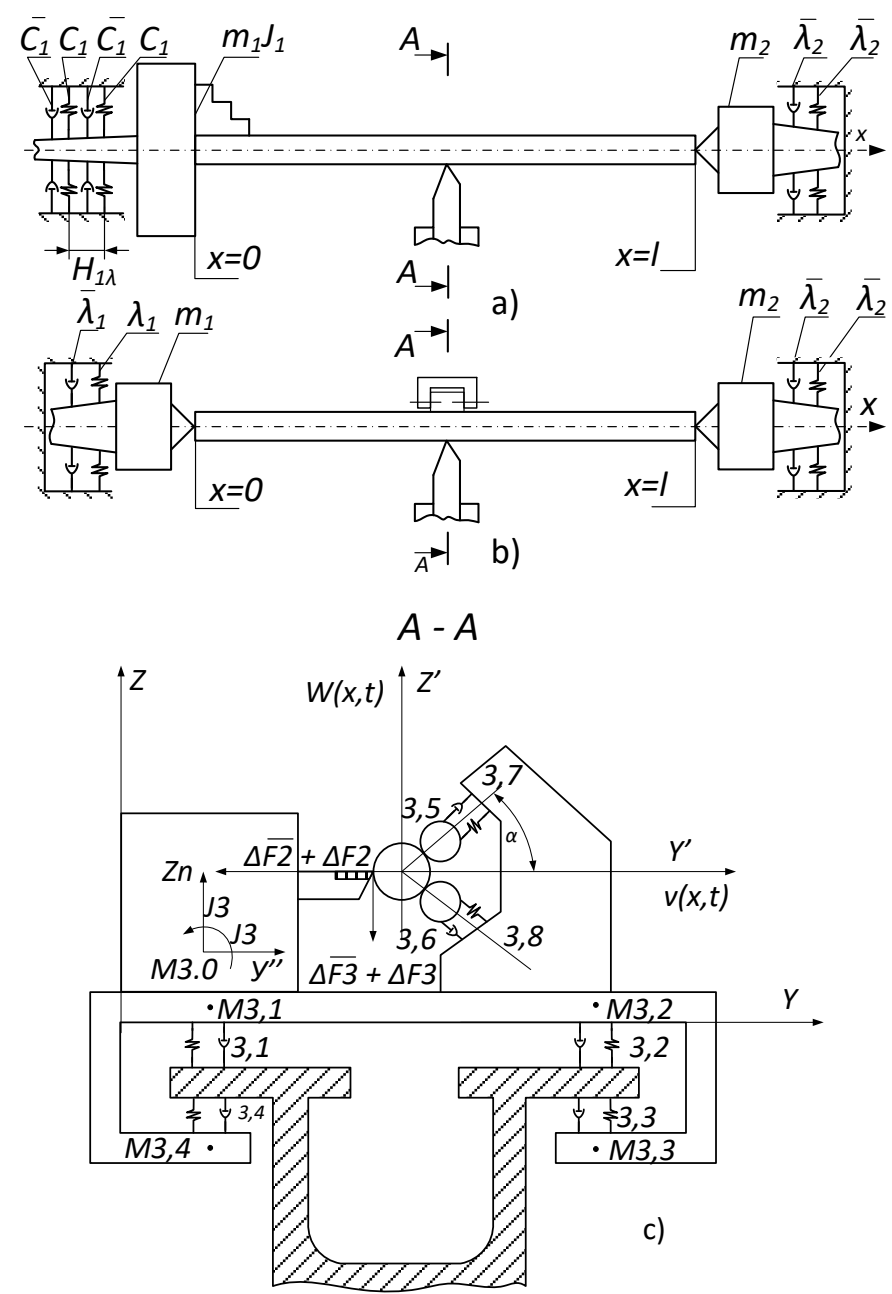

Fig. 1. Design diagrama, to obtain the oscillation equations of motion dominating the elastic elements of the considered machine tool system at various ways of shaft setting: a - processing in a selfcentering chuck with snap-action rear center; $\mathrm{b}, \mathrm{c}-$ treatment in centers using a vibration-damper lunette.

The design diagram shows two characteristic types of attachment of the left end of the shaft being processed - in the chuck and in the center. Thus rigidity installed in the spindle center is simulated in the form of two springs with stiffness $\lambda_{1}$ oriented in the direction of the 
axes $y$ and $z$. The energy dissipation in the center is denoted by the damping parameter $\bar{\lambda}$. In the case of fastening the shaft in the chuck, the design diagrama takes into account the stiffness and damping in the bearings of the spindle $\left(C_{1}, \overline{C_{1}}\right)$ and the distance between the bearings $H_{11}$.

The reduced mass of the moving parts of the front center (or chuck) denoted $m_{1}$, and the moment of inertia about its main inertia axis parallel to the axes $y$ and $z$-designated $J_{l}$. The right end of the shaft (section $x=l$ ) is attached in the rear rotating center, whose parameters are indicated in the design diagram with $m_{2}, \lambda_{2}, \overline{\lambda_{2}}$.

The second dominant vibrational element of the elastic system of the machine is a saddle with a slide. Due to the nature of processing of non-rigid shaft to provide the required accuracy and quality of the treated surface of a support the lunette-vibration damperis mounted on the saddle slide. (Fig. 2).

The oscillations of the saddle and the slide with the lunette, as a rule, take place in a plane perpendicular to the $x$ axis.

The equivalent mechanical model describing the vibrations of this node includes the mass $m_{3}$, the moment of inertia $J_{3}$ relative to the central axis of inertia $x^{\prime \prime}$, the stiffness $\lambda_{3 j}$ and damping coefficients $\overline{\lambda_{3 j}}, j=1,4$ joints, and the stiffness and damping of the contact elements of the lunette vibration damper $\lambda_{3.5}, \lambda_{3.6}, \overline{\lambda_{3.5}}, \overline{\lambda_{3.6}}$.

It sets out the equivalent mechanical model gives a fairly complete picture of the vibrational properties of the main elements of the dominant machine in view of various technological adjustments.

It should be noted that the mathematical model corresponding to this design diagrama should be a fairly complex system of partial differential equations with boundary conditions in the shaft sections $x=0, x=x_{0}, x=l$.

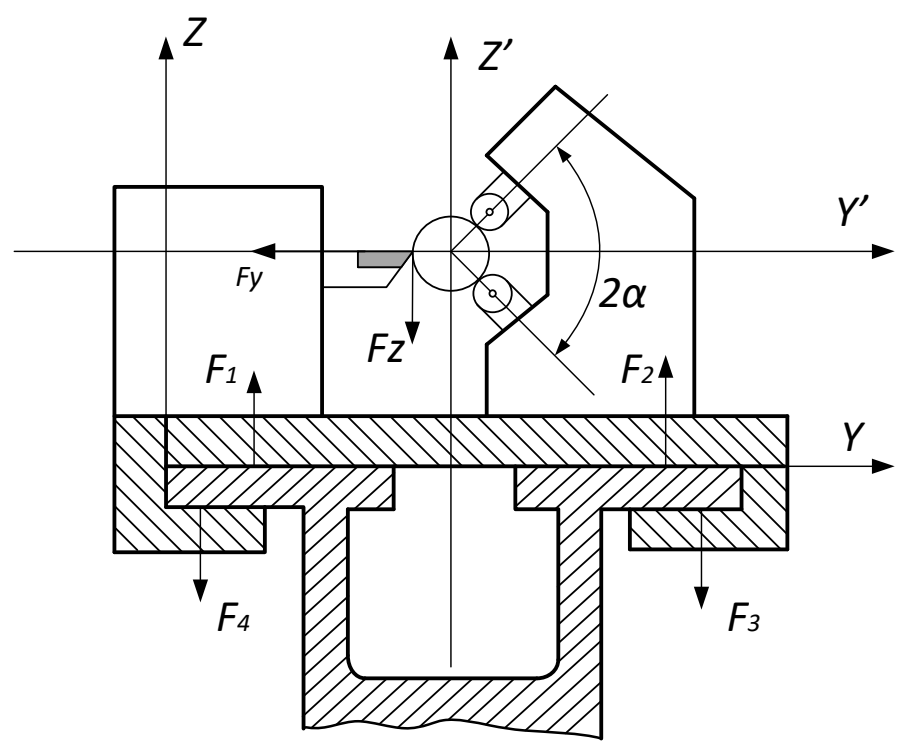

Fig. 2. Schematic of the processing of the shaft with a chisel using a vibration damper-lunette.

In accordance with the idealization adopted, the equations of motion of the EES machine's for processing non-rigid shafts are a system of two fifth-order partial differential equations describing transverse oscillations of a shaft with boundary conditions and two ordinary secondorder differential equations describing saddle oscillations. 
The shaft is assumed to be isotropic, it is assumed that when it oscillates, the hypothesis of flat sections is satisfied.

Under these conditions, the differential equations describing the oscillations of the processed shaft are reduced to the form:

$$
\begin{aligned}
& E J \frac{\partial^{4} V(x, t)}{\partial x^{4}}+\bar{h} E J \frac{\partial^{5} V(x, t)}{\partial x^{4} \partial t}+\rho S \frac{\partial^{2} V(x, t)}{\partial t^{2}}=\Delta F_{y} \delta\left(x-x_{0}\right), \\
& E J \frac{\partial^{4} W(x, t)}{\partial x^{4}}+\bar{h} E J \frac{\partial^{5} W(x, t)}{\partial x^{4} \partial t}+\rho S \frac{\partial^{2} W(x, t)}{\partial t^{2}}=\Delta F_{z} \delta\left(x-x_{0}\right),
\end{aligned}
$$

where $V(x, t)$ and $W(x, t)$ - the shaft bias in the horizontal and vertical planes respectively;

$\rho$ - the density of the material;

$S$ - the area of shaft cross-section;

$\bar{h}$ - the internal friction coefficient;

$E$ - the modulus of elasticity;

$J$ - the axial moment of inertia of shaft cross-section;

$\Delta F_{y}, \Delta F_{z}$ - the increments of cutting forces acting on the machined shaft, and the reactions of the support holder contact elements;

$\delta\left(x-x_{0}\right)$ - delta function characterizing point cutter application.

When deriving equations of shaft movements we consider temporarily that the saddle with the lunette does not oscillate.

Consider the left end of the shaft.

Depending on the method of attachment (in the center or chuck) two types of boundary conditions are possible here.

In the case of a shaft fixed at the centers the boundary condition at $x=x_{0}$ will be:

$$
\begin{gathered}
m_{1} \frac{\partial^{2} V(o, t)}{\partial t^{2}}=-\lambda_{1} V(o, t)-\bar{\lambda}_{1} \frac{\partial V(o, t)}{\partial t}-E J \frac{\partial^{3} V(o, t)}{\partial x^{3}}-h E J \frac{\partial^{4} V(o, t)}{\partial x^{3} \partial t}, \\
m_{1} \frac{\partial^{2} W(o, t)}{\partial t^{2}}=-\lambda_{1} W(o, t)-\bar{\lambda}_{1} \frac{\partial W(o, t)}{\partial t}-E J \frac{\partial^{3} W(o, t)}{\partial x^{3} \partial t}-\bar{h} E J \frac{\partial^{4} W(o, t)}{\partial x^{3} \partial t}, \\
E J \frac{\partial^{2} V(o, t)}{\partial x^{4}}+\bar{h} E J \frac{\partial^{3} V(o, t)}{\partial x^{2} \partial t}=0 \\
E J \frac{\partial^{2} W(o, t)}{\partial x^{2}}+\bar{h} E J \frac{\partial^{3} W(o, t)}{\partial x^{2} \partial t}=0
\end{gathered}
$$

where

$$
\begin{aligned}
& \lambda_{1}=\frac{C_{1} \lambda_{1}}{C_{1}+\lambda_{1}}, \\
& \overline{\lambda_{1}}=\frac{\overline{C_{1} \lambda_{1}}}{\overline{C_{1}}+\overline{\lambda_{1}}} .
\end{aligned}
$$


The first two equations represent the equality of the inertial forces to the elastic, dissipative, and shear forces at the end of the shaft. Two other relations describe zero equality of the moments at the end of the shaft.

When fixing the shaft in the chuck, $(x=0)$ the boundary conditions are written in the form:

$$
\begin{gathered}
m_{1} \frac{\partial^{2} V(o, t)}{\partial t^{2}}=-\lambda_{1} V(o, t)-\bar{\lambda}_{1} \frac{\partial V(o, t)}{\partial t}-E J \frac{\partial^{3} V(o, t)}{\partial x^{3}}-\bar{h} E J \frac{\partial^{4} V(o, t)}{\partial x^{3} \partial t} \\
m_{1} \frac{\partial^{2} W(o, t)}{\partial t^{2}}=-\lambda_{1} W(o, t)-\bar{\lambda}_{1} \frac{\partial W(o, t)}{\partial t}-E J \frac{\partial^{3} W(o, t)}{\partial x^{3}}-\bar{h} E J \frac{\partial^{4} W(o, t)}{\partial x^{3} \partial t} \\
J_{1} \frac{\partial^{3} V(o, t)}{\partial x \partial t^{2}}=-C_{1} \frac{\partial V(o, t)}{\partial x}-\bar{C}_{1} \frac{\partial^{2} V(o, t)}{\partial x \partial t}-E J \frac{\partial^{2} V(o, t)}{\partial x^{2}}-\bar{h} E J \frac{\partial^{3} V(o, t)}{\partial x^{2} \partial t} \\
J_{1} \frac{\partial^{3} W(o, t)}{\partial x \partial t^{2}}=-C_{1} \frac{\partial W(o, t)}{\partial x}-\bar{C}_{1} \frac{\partial^{2} W(o, t)}{\partial x \partial t}-E J \frac{\partial^{2} W(o, t)}{\partial x^{2}}-\bar{h} E J \frac{\partial^{3} W(o, t)}{\partial x^{2} \partial t}
\end{gathered}
$$

where

$$
\overline{C_{2}}=\overline{C_{2}} \cdot H_{1.1}^{2}, C_{1}=C_{1} \cdot H_{1.1}^{2}
$$

Comparing equation (3) and (4) it should be noted that the boundary conditions on the right end of the shaft $(x=l)$ differ only in the signs of the shaft shear forces:

$$
\begin{gathered}
m_{1} \frac{\partial^{2} V(l, t)}{\partial t^{2}}=-\lambda_{2} V(l, t)-\bar{\lambda}_{2} \frac{\partial V(l, t)}{\partial t}+E J \frac{\partial^{3} V(l, t)}{\partial x^{3}}-\bar{h} E J \frac{\partial^{4} V(l, t)}{\partial x^{3} \partial t}, \\
m_{1} \frac{\partial^{2} W(l, t)}{\partial t^{2}}=-\lambda_{2} W(l, t)-\bar{\lambda}_{2} \frac{\partial W(l, t)}{\partial t}+E J \frac{\partial^{3} W(l, t)}{\partial x^{3}}+\bar{h} E J \frac{\partial^{4} W(l, t)}{\partial x^{3} \partial t}, \\
E J \frac{\partial^{2} V(l, t)}{\partial x^{4}}+\bar{h} E J \frac{\partial^{3} V(l, t)}{\partial x^{2} \partial t}=0 \\
E J \frac{\partial^{2} W(l, t)}{\partial x^{4}}+\bar{h} E J \frac{\partial^{3} W(l, t)}{\partial x^{2} \partial t}=0 .
\end{gathered}
$$

In the cutting zone $\left(x=x_{0}\right)$ operates static cutting force, which is balanced by elastic forces of the shaft and the contact elements backrest reactions.

When the shaft oscillates, the technological parameters of cutting change, in particular the depth of cutting, as a result of which the dynamic components of the cutting force $\Delta F\left(\Delta F_{1}, \Delta F_{2}, \Delta F_{3}\right)$ and the reaction of the contact elements $\Delta F\left(\Delta \overline{F_{1}}, \Delta \overline{F_{2}}, \Delta \overline{F_{3}}\right)$ of the lunette start acting on the shaft (Pic. 1c).

From the design diagrama it follows that the point $x=x_{0}$ on the oscillating shaft, forces $F_{1}, F_{2}$ and $F_{3}$, related by the following relations:

$$
F_{1}=\Delta F_{1} ; F_{2}=\Delta F_{2}+\Delta \overline{F_{2}} ; F_{3}=\Delta F_{3}+\Delta \overline{F_{3}} .
$$




$$
\begin{gathered}
\Delta \overline{F_{2}}=-\left(V\left(x_{0}, t\right) \cdot \cos \alpha+W\left(x_{0}, t\right) \cdot \sin \alpha\right) \cdot \lambda_{3.5} \cdot \cos \alpha-\left(V\left(x_{0}, t\right) \cdot \cos \alpha-\right. \\
\left.-W\left(x_{0}, t\right) \cdot \sin \alpha\right) \cdot \lambda_{3.6} \cdot \cos \alpha \\
\Delta \bar{F}_{3}=-\left(V\left(x_{0}, t\right) \cdot \cos \alpha+W\left(x_{0}, t\right) \cdot \sin \alpha\right) \cdot \lambda_{3.5} \cdot \sin \alpha+\left(V\left(x_{0}, t\right) \cdot \cos \alpha-\right. \\
\left.-W\left(x_{0}, t\right) \cdot \sin \alpha\right) \cdot \lambda_{3.6} \cdot \sin \alpha .
\end{gathered}
$$

\section{Conclusion}

The following results were obtained during the research:

1. The selection of the main dominant vibrational elements of the elastic system of the machine tool during processing of non-rigid shafts is substantiated.

2. To study the vibration resistance of nonrigid shafts, an equivalent mechanical model of the elastic system of the machine tool was developed.

3. Obtained boundary conditions at the ends of the shaft and in the cutting zone, considering the use of the species of technological equipment of the machine.

Through the developed mechanical model the authors of the article investigated the boundaries of the stability regions for various types of technological equipment and revealed the patterns of the influence of various technological adjustments on the stability of the dynamic system of machine tools [14,15], which, on the basis of the analysis, will make important practical recommendations.

\section{References}

1. S.L. Vasilevykh, L.A Vasilevykh, The study of the macro and micro forming of a low rigidity parts in the machining area extending beyond the material elasticity, 350 (Dnepropetrovsk, 2009).

2. S.L. Vasilevykh, V.E. Saitov, Features of processing of nonrigid shafts. Modern science-intensive technologies, 67-68 (№ 11, 2012).

3. S.L. Vasilevykh, V.E. Saitov, Technological means for ensuring the vibration resistance of the process of turning non-rigid shafts. Modern high technology, 7-10 (№3, 2014).

4. S.L. Vasilevykh, All-Russian annual scientific and practical conference Society, Innovations: Sat. Materials, Vyatka State University, 1556-1560 (Science, Kirov, 2014).

5. X. Han, H. Ouyang, M. Wang, Y. Mao, N. Hassan, Selfexcited vibration of workpieces in a turning process, Proceedings of the Institution of Mechanical Engineers, 19581970 (Journal of Mechanical Engineering Science, № 226(8s), 2012).

6. C.F. Bisu, P. Darnis, A. Gérard, J-Y K'nevez, Displacements analysis of self-excited vibrations in turning, The International Journal of Advanced Manufacturing Technology, 1-16 (№ 44(1s), 2008).

7. C.F. Bisu, A. Gérard, J-Y K'nevez, R. Laheurte, O. Cahuc, Self-excited vibrations in turning: Forces torsor analysis, The International Journal of Advanced Manufacturing Technology, 447-462 (№ 44, 2009).

8. Yu.I. Gorodetsky, V.Ya. Prodiu, Experimental determination of the dynamic characteristics of the cutting process with the aid of a computer. Machines and tools, 25-27 (№6, 1980). 
9. Yu.I. Gorodetsky, A.S. Budankov, V.N. Komarov, On a system of the experimental study of the dynamics in the process of cutting metals, Problems of mechanical engineering and reliability of machines, 80-86 (RAS, 1, 2008).

10. O.A. Yamnikova, Construction of mathematical models of non-rigid shaft vibrations in cutting, STIN, 18-21 (Moscow, 1, 2003).

11. Yu.I. Gorodetsky, Creation of mathematical models of complex self-oscillating systems in machine-tool construction Automation of design, Mechanical Engineering, 1986. P. 203-220.

12. Yu.F. Kopelev, Influence of parameters of machine subsystems on its vibration resistance, Metal-cutting machine tools, 1983. - Vol. 11. - P. 12-17.

13. Elyasberg M.E., On the independence of the boundary of stability of the process of cutting from a perturbation in the trace, Machines and Tools, 1976. - Vol. 11. - P. 3236.

14. S. Vasilevykh, V. Buravlev, E. Shelihov, Method of mathematical model development to study vibration resistance of non-rigid shaft linear turning, Procedia Engineering, International Conference on Industrial Engineering (ICIE 2017), 2017. - Vol. 206. - P. 373-379.

15. S.L. Vasilevykh, E.S. Shelihov, Research of vibration resistance of non-rigid shafts turning with various technological set-ups, MATEC Web of Conferences, proceedings of the International Conference on Modern Trends in Manufacturing Technologies and Equipment (ICMTMTE 2017), Vol. 129. - 5 p. (Sevastopol, 2017). 